\title{
Multidrug-Resistant Tuberculosis in Côte d'Ivoire from 1995 to 2016: Results of National Surveys
}

\author{
Kouassi N'Guessan ${ }^{1 *}$, Timothée Ouassa ${ }^{2}$, Anna S. Dean ${ }^{3}$, Riccardo Alagna $^{4}$, Guy Damien Adagra ${ }^{5}$, \\ Valeri Ibode $^{5}$, Daniela M. Cirillo ${ }^{4}$ and Jacquemin Kouakou ${ }^{5}$ \\ ${ }^{1}$ Laboratoire National de Référence de la Tuberculose, Institut Pasteur de Côte d'Ivoire \\ ${ }^{2}$ Centre de Diagnostic et de Recherche sur le Sida, Abidjan, Côte d'Ivoire \\ ${ }^{3}$ World Health Organization, Global TB Programme, Geneva, Switzerland \\ ${ }^{4}$ TB Supranational Reference Laboratory, IRCCS San Raffaele Scientific Institute, Milan, Italy \\ ${ }^{5}$ Programme National de Lutte contre la Tuberculose, Côte d'Ivoire
}

Received: 24 December 2017; accepted: 16 March 2018

\begin{abstract}
Setting: Tuberculosis (TB) drug resistance survey was conducted in 2016-2017 to estimate the burden of drug-resistant TB in Côte d'Ivoire.

Design: A cross-sectional cluster-based survey was conducted. All eligible smear positive patients were interviewed using a structured questionnaire to collect clinical and sociodemographic information and tested by the Xpert Mycobacterium tuberculosis/rifampicin (MTB/RIF) assay. If resistant to rifampicin, solid and liquid cultures were performed. Phenotypic drug susceptibility testing (DST) was conducted in liquid medium for rifampicin, isoniazid, ethambutol, streptomycin, ofloxacin, and amikacin.

Results: Of the 1105 sputum smear positive patients enrolled, 995 new and 100 previously treated patients were positive for Mycobacterium tuberculosis complex by Xpert. Proportion of patients with rifampicin resistance was $4.6 \%(95 \%$ CI: $2.4-6.7)$ and $22 \%$ (95\% CI: 13.7-30.3), respectively, for new and previously treated patients. Second-line DST results were available for most rifampicin-resistant patients. None were resistant to amikacin, only two were ofloxacin-resistant. Apart from the antecedent of previously treatment for TB, no other risk factors for rifampicin resistance were detected. Conclusion: Prevalence of rifampicin resistance among TB patients in Côte d'Ivoire is higher than that in other countries in the region. Surveillance of drug resistance, through an expanded GeneXpert network, and programmatic management of drug-resistant TB (PMDT) must be strengthened in Côte d'Ivoire.
\end{abstract}

Keywords: Tuberculosis, survey, prevalence, drug resistance, Xpert MTB/RIF

\section{Introduction}

Antimicrobial resistance threatens global health and security. In 2014, the World Health Assembly called for the international community to take action against antimicrobial resistance [1]. Development of drug-resistant Mycobacterium tuberculosis strains, especially multidrug-resistant tuberculosis (MDR-TB) due to resistance to the two most powerful first-line anti-tuberculosis (TB) drugs, isoniazid and rifampicin, poses a major challenge to the control of TB worldwide $[2,3]$.

Guidelines recommended by the World Health Organization (WHO) to diagnose, treat, and prevent TB have been implemented in Côte d'Ivoire since 1995 [4-6]. There were an estimated 36,000 new cases of TB in 2016 [7]. The main method used for diagnosis of TB is sputum smear microscopy (SSM) based on Ziehl-Neelsen or Auramin staining of two sputum samples (spot and morning) on consecutive days.

Surveillance is a critical component of detecting, planning, and responding to the public health challenge of drug-resistant TB. As Côte d'Ivoire does not yet have the capacity to routinely test all TB patients for drug susceptibility, two national drug resistance surveys were conducted in 1995 and 2006. The proportions of new TB cases with MDR-TB in these surveys were 5.3\% (95\% CI: $3.1-8.4 \%$ ) and $2.5 \%$ (95\% CI: $1.1-4.9 \%$ ), respectively $[8,9]$. The third national survey was conducted in 2016 under rou-

\footnotetext{
* Corresponding author: N'GUESSAN Kouasssi Raymond; E-mail: kouassinguessan@ pasteur.ci,ngueskr@gmail.com
}

tine programmatic conditions to provide updated data on the burden of drug-resistant TB.

\section{Methods}

Study Design. This cross-sectional survey targeted all consecutively notified new and previously treated sputum smearpositive pulmonary TB diagnosed in the country, including both adults and children. The survey was implemented using a cluster design, with 35 clusters selected from a list of all of the $230 \mathrm{~TB}$ diagnostic centres in the country using a probability-proportionalto-size approach. These 35 clusters were distributed across 28 diagnostic centres. The target sample size was 971 new patients, with previously treated patients being enrolled opportunistically until the sample size for new patients was reached. The enrolment period was 12 months from 1 March 2016 to 28 February 2017. A questionnaire was administered by a trained interviewer at the time of enrolment, which included questions relating to demographics, TB treatment history (new or previously treated), social factors (education level, previous incarceration), and other health indicators (history of smoking, human immunodeficiency virus [HIV] status). Ethical clearance for the survey was obtained from the National Ethics Committee of Côte d'Ivoire.

Laboratory Methods. Two sputum specimens (spot and morning samples) were collected from all eligible patients.

Sputum smear microscopy was performed after Auramin staining, as per national guidelines for routine TB diagnosis. One sputum sample from each sputum smear-positive patient was tested by Xpert Mycobacterium tuberculosis/rifampicin (MTB/RIF) (Cepheid,

This is an open-access article distributed under the terms of the Creative Commons Attribution-NonCommercial 4.0 International License (https://creativecommons.org/licenses/by-nc/4.0/), which permits unrestricted use, distribution, and reproduction in any medium for non-commercial purposes, provided the original author and source are credited, a link to the CC License is provided, and changes - if any - are indicated. 
CA, USA) for the identification of the M. tuberculosis complex (MTBC) and the determination of rifampicin susceptibility status, either at the peripheral diagnostic centre or in the capital, Abidjan, at the laboratories of the Institut Pasteur or the Centre de Diagnostic et de Recherche sur le SIDA. All samples with resistance to rifampicin were transported on ice to the central laboratories in Abidjan for culture and phenotypic drug susceptibility testing (DST), as well as a randomly selected $10 \%$ of rifampicin-susceptible samples as a control.

Handling of sputum for culture was performed in a Class II biosafety cabinet in a biosafety level 3 (BSL3) laboratory. Sputum samples were decontaminated by the standard $N$-acetyl-1cysteine $-4 \% \mathrm{NaOH}$ procedure. The supernatant was discarded, and the pellet was resuspended with sterile phosphate buffer to a final volume of $2 \mathrm{~mL}$. This was inoculated into one BACTEC Mycobacteria Growth Indicator Tube (MGIT) 960 tube $(0.5 \mathrm{~mL})$ and two Lowenstein-Jensen slants $(0.2 \mathrm{~mL}$ each). Prior to inoculation, the BACTEC MGIT 960 media were supplemented with an antibiotic mixture containing polymyxin B, amphotericin B, nalidixic acid, trimethoprim, and azlocillin (PANTA) and growth supplement (Becton Dickinson). BACTEC MGIT 960 tubes were incubated at $37{ }^{\circ} \mathrm{C}$ in the BACTEC MGIT 960 instrument, in which they were automatically monitored each hour for fluorescence development for 42 days or until a positive signal developed. Solid media were incubated at $37{ }^{\circ} \mathrm{C}$ for 8 weeks and were inspected weekly or until mycobacterial colonies were seen. Cultures were considered positive only after detection of MTBC by the immunochromatographic assay, SD BIOLINE TB Ag MPT64 (Standard Diagnostics, Seoul, South Korea). This assay is rapid, simple, and reliable with a demonstrated high sensitivity and specific on clinical isolates $[10,11]$.

Phenotypic DST with the automated MGIT system was performed on MTBC positive cultures according to the manufacturer's instructions using the BD BACTEC MGIT SIRE drug kit (Becton, Dickinson \& Company, Sparks, MD, USA). The DST assays were performed at the following concentrations: $0.1 \mathrm{mg} / \mathrm{L}$ for isoniazid, $1.0 \mathrm{mg} / \mathrm{L}$ for rifampicin, $1.0 \mathrm{mg} / \mathrm{L}$ for streptomycin, and $5.0 \mathrm{mg} / \mathrm{L}$ for ethambutol. For rifampicin-resistant isolates, phenotypic DST was also performed for amikacin and ofloxacin at concentrations of $1.0 \mathrm{mg} / \mathrm{L}$ and $2.0 \mathrm{mg} / \mathrm{L}$, respectively. Phenotypic DST tubes were inoculated with $0.5 \mathrm{~mL}$ of MGIT culture. A drug-free control was also inoculated with $0.5 \mathrm{~mL}$ of a $1: 100$ dilution of positive culture broth in sterile saline. The tubes were incubated in the BACTEC MGIT instrument. The H37rv strain was used for internal quality control of phenotypic DST.

Statistical Analysis. Data were entered into a pre-designed EpiData Entry 3.1 software (EpiData Association, Odense, Denmark) and analysed using Stata version 14.0 (StatCorp, Texas, USA). Proportions of new and previously treated cases with rifampicin resistance and MDR-TB with 95\% confidence intervals $(95 \% \mathrm{CI})$ were computed using logistic regression with robust standard errors to account for clustering. The analysis was performed both with and without sample weights (target cluster size divided by the actual number enrolled in cluster) to account for under- and over-enrolment, and the results were compared. For samples with a rifampicin result from both Xpert MTB/RIF and phenotypic DST, the level of interrater agreement was assessed by the Kappa test.

\section{Results}

Patient Inclusion. A total of 1002 new sputum smear positive pulmonary TB patients were enrolled, corresponding to an enrolment rate of $103 \%$. Additionally, 103 previously treated patients were enrolled across the 35 clusters (Figure 1). The median age of patients was 33 years (range 15-80). Males represented $65.5 \%$ of the patients, and $12 \%$ were HIV-positive (Table 1). An Xpert MTB/RIF assay result was available for 1104 cases. Of these, 1095 were positive for MTBC, including 995 new and 100 previously treated cases. The concordance between smear microscopy and GeneXpert for bacteriological confirmation was $99.2 \%$.

Drugs Susceptibility Testing Results. Rifampicin resistance was detected by Xpert MTB/RIF in 68 patients, representing

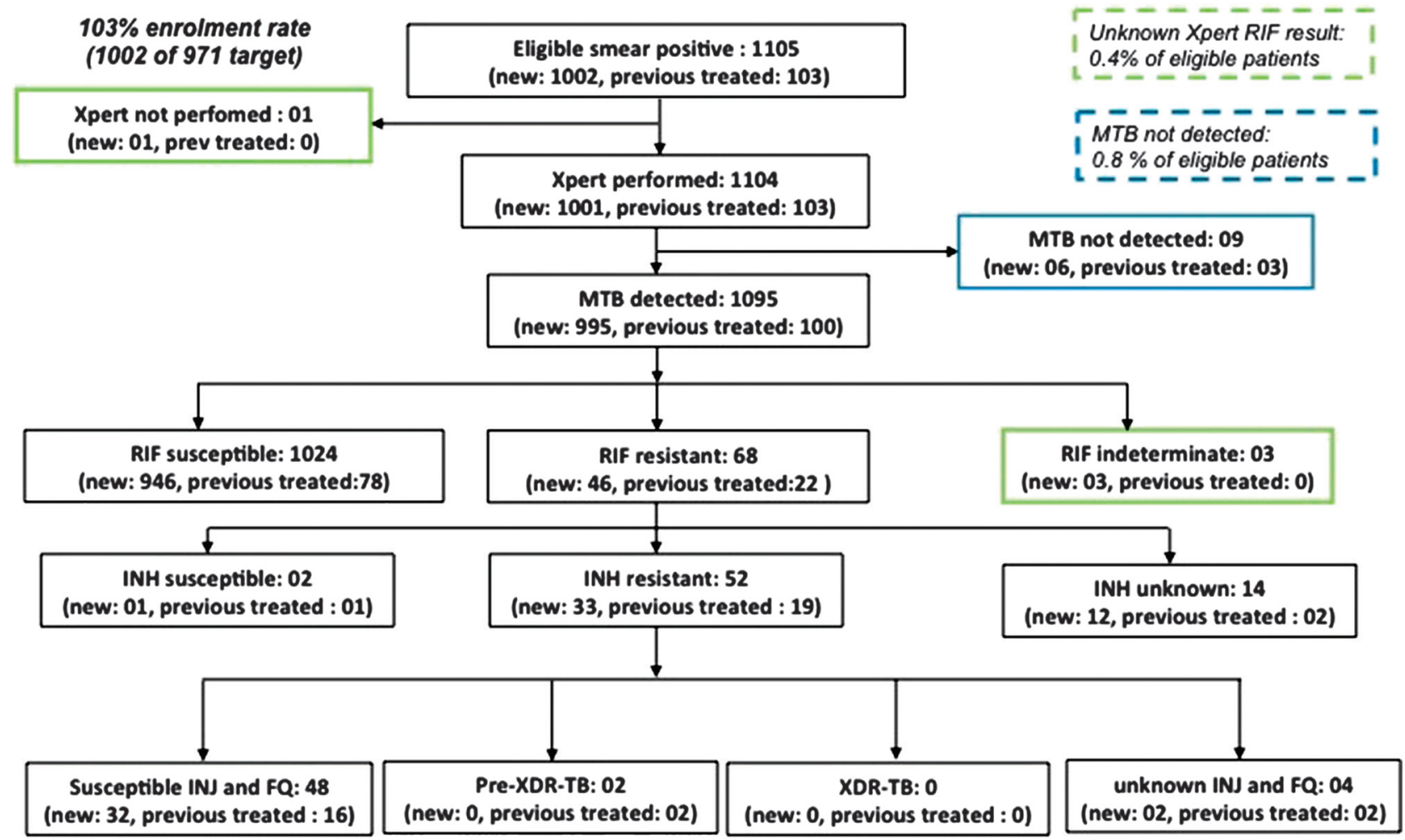

Figure 1. Flowchart of patients enrolled $(\mathrm{FQ}=$ fluoroquinolones; $\mathrm{INH}=$ isoniazid; $\mathrm{INJ}=$ second-line injectable agents; RIF = rifampicin; $\mathrm{MTB}=$ Mycobacterium tubercolosis complex; XDR-TB: Extensively drug-resistant tuberculosis) 
Table 1. Characteristics of GeneXpert MTB-positive patients included in the national anti-TB drug resistance survey

\begin{tabular}{lc}
\hline Characteristic & $\begin{array}{c}\text { Number of patients } \\
(n=1095)\end{array}$ \\
\hline History of treatment & $995(90.9 \%)$ \\
New TB cases & $100(9.1 \%)$ \\
Previously treated & \\
Sex & $715(65.3 \%)$ \\
Male & $380(34.7 \%)$ \\
Female & \\
Age & $15-80$ years \\
Range & 33 years \\
Median & \\
HIV status & $958(87.5 \%)$ \\
Negative & $130(12 \%)$ \\
Positive & $7(0.6 \%)$ \\
Unknown &
\end{tabular}

46 new patients and 22 previously treated patients. The proportion of new TB cases with rifampicin resistance was $4.6 \%$ (95\% CI: 2.4-6.7), with 22\% (95\% CI: 13.7-30.3) among previously treated cases. Due to most clusters reaching their target sample size, sample weights had minimal impact on the results and were not used in final analysis.

Of the 68 cases with rifampicin resistance detected by Xpert MTB/RIF, cultures were performed for only 58, due to challenges in sample transport. Of these, two cultures were contaminated, and two were negative. Phenotypic DST was therefore successfully performed for 54 Xpert MTB/RIF rifampicinresistant patients (Table 2). Discordant results between Xpert MTB/RIF and phenotypic drug susceptibility testing in liquid medium were observed for 8 of the 54 isolates (14.8\%) (all were rifampicin-resistant by Xpert MTB/RIF but rifampicin-susceptible by phenotypic testing). DST was performed for 114 Xpert MTB/RIF rifampicin-susceptible samples of which 112 phenotypic DST results were available.

Six DST results were not interpretable, 2 for rifampicin (Xpert rifampicin-susceptible), 1 in new and previous traited patients (Table 1). The 4 others were for second line drug (Figure 1 and Table 2)

Overall, the level of agreement between Xpert MTB/RIF and phenotypic DST for the 168 samples for which results were available by both tests was $94 \%$ (kappa $=0.859)$.
Among 68 samples with rifampicin resistance on Xpert MTB/ RIF, an isoniazid result was available for 54 (79\%). Most of these (96\%) were MDR-TB. Fifty of these 54 patients also had phenotypic DST results available for second-line drug susceptibility testing. No resistance was detected to second-line injectable drugs and resistance to ofloxacin was only detected in two patients.

Given that isoniazid susceptibility testing was only conducted among rifampcin-resistant cases, for which results were missing for $20 \%$, as well as a subset of rifampicin-susceptible cases, statistical multiple imputations of missing values could not be performed. Minimum and maximum estimations of MDR-TB were calculated by assuming that all of the rifampicin-resistant cases missing an isoniazid result were either isoniazid-susceptible or isoniazid-resistant, respectively. Minimum estimations were 3.3\% (95\% CI: $1.9-4.8 \%$ ) in new and 19.2 (95\% CI: $10.8-27.6 \%)$ in previously treated cases. Maximum estimations were $4.5 \%$ (95\% CI: $2.4-6.7 \%)$ in new and $21.2 \%(95 \%$ CI: $13.0-29.4)$ in previously treated cases (Table 3).

Risks Factors For Rifampicin Resistance. No associations were found between rifampicin resistance and age, sex, alcohol consumption, history of incarceration, or smoking. Patients who had been previously treated for TB had odds of rifampicinresistant TB that was $6.0(95 \% \mathrm{CI}: 3.4-10.7)$ times higher than new cases $(p<0.001)$.

\section{Discussion}

In this manuscript, we report the results of the third national anti-TB drug resistance survey conducted in Côte d'Ivoire. High quality clinical, laboratory, and sociodemographic data collected from TB patients allowed a national representative analysis of patient characteristics, drug resistance patterns, and risk factors for resistance. With $4.6 \%$ of new and $22 \%$ of previously treated TB cases being infected by rifampicin-resistant strains, the burden of drug-resistant TB in Côte d'Ivoire is notably higher than in other countries in West Africa, such as Burkina Faso (2.1\% rifampicin resistance in new and $14.3 \%$ in previously treated cases in 2017), Ghana $(1.5 \%$ rifampicin resistance in new and $7 \%$ in previously treated cases in 2017), or Senegal (0.5\% MDR-TB in new and $17.5 \%$ in previously treated cases in 2014). However, at the regional level, the prevalence of drug resistance among TB patients

Table 2. DST pattern of clinical isolates

\begin{tabular}{|c|c|c|c|}
\hline GeneXpert MTB/RIF assay results & Drug resistance pattern & $\begin{array}{l}\text { New cases } \\
(n=130)\end{array}$ & $\begin{array}{l}\text { Previous treated } \\
\text { patients }(n=32)\end{array}$ \\
\hline \multirow[t]{4}{*}{ MTB detected and rifampicin susceptible } & $\mathrm{RIF}^{\mathrm{S}}+\mathrm{INH}^{\mathrm{S}}+\mathrm{STR}^{\mathrm{S}}+\mathrm{EMB}^{\mathrm{S}}+\mathrm{AMK}^{\mathrm{S}}+\mathrm{OFX}^{\mathrm{S}}$ & 85 & 7 \\
\hline & $\mathrm{RIF}^{\mathrm{S}}+\mathrm{INH}^{\mathrm{S}}+\mathrm{STR}^{\mathrm{R}}+\mathrm{EMB}^{\mathrm{S}}+\mathrm{AMK}^{\mathrm{S}}+\mathrm{OFX}^{\mathrm{S}}$ & 1 & 4 \\
\hline & $\mathrm{RIF}^{\mathrm{S}}+\mathrm{INH}^{\mathrm{R}}+\mathrm{STR}^{\mathrm{S}}+\mathrm{EMB}^{\mathrm{S}}+\mathrm{AMK}^{\mathrm{S}}+\mathrm{OFX}^{\mathrm{S}}$ & 8 & 2 \\
\hline & $\mathrm{RIF}^{\mathrm{S}}+\mathrm{INH}^{\mathrm{R}}+\mathrm{STR}^{\mathrm{R}}+\mathrm{EMB}^{\mathrm{S}}+\mathrm{AMK}^{\mathrm{S}}+\mathrm{OFX}^{\mathrm{S}}$ & 4 & 1 \\
\hline \multirow[t]{8}{*}{ MTB detected and rifampcin resistant } & $\mathrm{RIF}^{\mathrm{S}}+\mathrm{INH}^{\mathrm{R}}+\mathrm{STR}^{\mathrm{R}}+\mathrm{EMB}^{\mathrm{S}}+\mathrm{AMK}^{\mathrm{S}}+\mathrm{OFX}^{\mathrm{S}}$ & 1 & 2 \\
\hline & $\mathrm{RIF}^{\mathrm{S}}+\mathrm{INH}^{\mathrm{R}}+\mathrm{STR}^{\mathrm{R}}+\mathrm{EMB}^{\mathrm{R}}+\mathrm{AMK}^{\mathrm{S}}+\mathrm{OFX}^{\mathrm{S}}$ & 5 & - \\
\hline & $\mathrm{RIF}^{\mathrm{R}}+\mathrm{INH}^{\mathrm{R}}+\mathrm{STR}^{\mathrm{S}}+\mathrm{EMB}^{\mathrm{S}}+\mathrm{AMK}^{\mathrm{S}}+\mathrm{OFX}^{\mathrm{S}}$ & 5 & 1 \\
\hline & $\mathrm{RIF}^{\mathrm{R}}+\mathrm{INH}^{\mathrm{R}}+\mathrm{STR}^{\mathrm{R}}+\mathrm{EMB}^{\mathrm{S}}+\mathrm{AMK}^{\mathrm{S}}+\mathrm{OFX}^{\mathrm{S}}$ & 5 & 5 \\
\hline & $\mathrm{RIF}^{\mathrm{R}}+\mathrm{INH}^{\mathrm{R}}+\mathrm{STR}^{\mathrm{R}}+\mathrm{EMB}^{\mathrm{R}}+\mathrm{AMK}^{\mathrm{S}}+\mathrm{OFX}^{\mathrm{S}}$ & 14 & 7 \\
\hline & $\mathrm{RIF}^{\mathrm{R}}+\mathrm{INH}^{\mathrm{R}}+\mathrm{STR}^{\mathrm{S}}+\mathrm{EMB}^{\mathrm{R}}+\mathrm{AMK}^{\mathrm{S}}+\mathrm{OFX}^{\mathrm{S}}$ & 2 & 1 \\
\hline & $\mathrm{RIF}^{\mathrm{R}}+\mathrm{INH}^{\mathrm{R}}+\mathrm{STR}^{\mathrm{S}}+\mathrm{EMB}^{\mathrm{R}}+\mathrm{AMK}^{\mathrm{S}}+\mathrm{OFX}^{\mathrm{R}}($ pre-XDR-TB $)$ & - & 1 \\
\hline & $\mathrm{RIF}^{\mathrm{R}}+\mathrm{INH}^{\mathrm{R}}+\mathrm{STR}^{\mathrm{R}}+\mathrm{EMB}^{\mathrm{S}}+\mathrm{AMK}^{\mathrm{S}}+\mathrm{OFX}^{\mathrm{R}}($ pre-XDR-TB $)$ & - & 1 \\
\hline
\end{tabular}

$\mathrm{RIF}^{\mathrm{S}}$ : rifampin susceptible; $\mathrm{RIF}^{\mathrm{R}}$ : rifampin resistant; $\mathrm{INH}^{\mathrm{S}}$ : isoniazid susceptible; $\mathrm{INH}^{\mathrm{R}}$ : isoniazid resistant; $\mathrm{STR}^{\mathrm{S}}$ : streptomycin susceptible; STR $^{\mathrm{R}}$ : streptomycin resistant; $\mathrm{EMB}^{\mathrm{S}}$ : ethambutol susceptible; $\mathrm{EMB}^{\mathrm{R}}$ : ethambutol resistant; $\mathrm{AMK}^{\mathrm{S}}$ : amikacin susceptible; $\mathrm{AMK}^{\mathrm{R}}$ : amikacin resistant; $\mathrm{OFX}^{\mathrm{S}}$ : ofloxacin susceptible; OFX ${ }^{\mathrm{R}}$ : ofloxacin resistant; XDR-TB: extensively drug-resistant TB.

Table 3. Prevalence of MDR-TB and rifampicin resistance from 1995 to 2016

\begin{tabular}{|c|c|c|c|c|}
\hline \multirow[t]{2}{*}{ Prevalences } & \multirow{2}{*}{$\frac{1995[8]}{\text { New cases }(n=320)}$} & \multirow{2}{*}{$\frac{2006[9]}{\text { New cases }(n=320)}$} & \multicolumn{2}{|c|}{2016} \\
\hline & & & New cases $(n=995)$ & Previously treated $(n=100)$ \\
\hline $\begin{array}{l}\text { Rifampicine resistance } \\
\text { MDR-TB }\end{array}$ & $\begin{array}{c}\text { Not applicable } \\
17(5.3 \%) \\
(95 \% \text { CI: } 3.1-8.4 \%)\end{array}$ & $\begin{array}{c}\text { Not applicable } \\
8(2.5 \%) \\
(95 \% \text { CI: } 1.1-4.9 \%)\end{array}$ & $\begin{array}{l}46 \text { (4.6\%) (95\% CI: } 2.4-6.8 \%) \\
\text { mE*: } 3.3 \% \text { (95\% CI: } 1.9-4.8 \%) \\
\text { ME: } 4.5 \% \text { (95\% CI: } 2.4-6.7 \%)\end{array}$ & $\begin{array}{c}22(22 \%)(95 \% \text { CI: } 13.7-30.3 \%) \\
\left.\mathrm{mE}^{*}: 19.2 \% \text { (95\% CI: } 10.8-27.6 \%\right) \\
\text { ME: } 21.2 \%(95 \% \text { CI: } 13.0-29.4 \%)\end{array}$ \\
\hline
\end{tabular}


in Africa is lower than in other parts of the world (regional estimate of $2.7 \%$ rifampicin resistance among new patients) [2].

Although the standard first-line anti-TB treatment regimen recommended by WHO (2RHZE/4RH) has been implemented since 2002, Côte d'Ivoire suffered a civil war from 2002 to 2011. It is possible that disruption to health services during this period may have negatively impacted on the control of TB and facilitated the emergence of resistant strains. Prior to 2012, there were no national guidelines for programmatic management of drugresistant TB (PMDT), including DST among presumptive MDR-TB cases or provision of an appropriate treatment regimen for drug-resistant patients. This may have allowed ongoing transmission of drug-resistant strains in Côte d'Ivoire. Encouragingly, resistance to second-line drugs among rifampicin-resistant strains is very low which means that most of these patients would be eligible for the shorter MDR-TB treatment regimen of 9-12 months [12]. There is ongoing investment in strengthening PMDT, with 27 MDR-TB treatment sites currently across the country.

Not unsurprisingly, a history of previous treatments for TB was significantly associated with rifampicin resistance. In this survey, 11 of the 22 rifampicin-resistant previously treated patients were the cases who had failed a treatment course. According to national guidelines, treatment failures are identified only 5 months after treatment initiation. This poses a risk for the transmission of drug-resistant strains in the community. The WHO's End TB Strategy calls for universal DST for all TB patients at the time of treatment initiation [13]. The ongoing expansion of the GeneXpert network in Côte d'Ivoire is allowing more patients to access rapid testing. There are currently 13 instruments across the country, and there is a plan to equip each of the 29 health districts in the country with an instrument by 2020 . This will facilitate prompter diagnosis of drug resistance and initiation of an appropriate treatment regimen. However, sputum transport networks, laboratory capacity and data management systems must also be strengthened in order to achieve the ultimate aim of establishing a continuous surveillance system for anti-TB drug resistance in Côte d'Ivoire.

Among the 54 rifampicin-resistant samples detected by Xpert MTB/RIF for which results were also available by phenotypic DST, discordance was noted in $14.8 \%$. This discordance may have been due to administrative errors such as sample mislabeling, or technical errors in conducting phenotypic DST. Furthermore, the BACTEC MGIT 960 system has been shown to miss some mutations in the $r p o B$ gene that are known to confer resistance to rifampicin [14]. Overall, among the 168 samples for which results were available by both genotypic and phenotypic tests, the level of agreement was high, reflecting the reliable performance of Xpert MTB/RIF compared to conventional methods.

There are some limitations to this survey. Firstly, the survey enrolled sputum smear positive patients only. However, there is no evidence that the prevalence of resistance differs between smear positive and smear negative patients [15]. Secondly, phenotypic DST for first and second-line drugs was only performed on sputum samples that were resistant to rifampicin by Xpert MTB/RIF. This means that other resistance patterns in the absence of rifampicin resistance could not be investigated. However, the use of this assay greatly simplified logistics. A rifampicin result was available for almost all of the enrolled patients, allowing an accurate estimation of the prevalence of rifampicin resistance among new and previously treated patients.

\section{Conclusion}

The third national anti-TB drug resistance survey in Côte d'Ivoire detected a higher prevalence of rifampicin resistance among TB patients than in neighbouring countries. Drug-resistant TB poses a major threat to reducing the burden of TB in Côte d'Ivoire. The surveillance of resistance must be strengthened through expansion of the GeneXpert network, coupled with improved access to appropriate care through PMDT.

\section{Funding Sources}

This study was funded by Global Fund to Fight against tuberculosis.

\section{Author Contributions}

K.N., T.O., G.D.A., D.C., and A.S.D. conceptualized and designed the study. A.S.D. and V.I. performed statistical analysis. K.N., J.K., A.S.D., and R.A. analyzed and interpreted the data. K.N. and R.A. supervised the study.

\section{Conflict of Interest}

The authors have no commercial associations or sources of support that might pose a conflict of interest.

\section{Disclaimer}

The findings and conclusions in this report are those of the authors and do not necessarily represent the official position of the National Institutes of Health.

Acknowledgments. The authors would like to thank all patients who were involved in this survey. We also wish to thank all chief doctors of TB centres and Dennis Falzon (World Health Organization, Global TB Programme, Geneva, Switzerland) for their support.

\section{References}

1. Resolution WHA67/25: antimicrobial resistance. Presented at the 67th World Health Assembly. Geneva, May 24, 2014 (Resolutions and Decisions; Annexes [WHASS1/2014WHA67/2014/REC/25]). Geneva, Switzerland; 2014.

2. World Health Organization. Global tuberculosis report 2017. WHO/HTM/ TB/2017.23. Geneva, Switzerland; 2017.

3. World Health Organization. Definitions and reporting framework for tuberculosis - 2013 revision. WHO/HTM/TB/2013.2. Geneva, Switzerland; 2013.

4. World Health Organization. What is DOTS? A Guide to Understanding the WHO-recommended TB Control Strategy Known as DOTS. WHO/CDS/CPC/TB/ 99.270. World Health Organization, Geneva, Switzerland; 1999.

5. World Health Organization. THE STOP TB STRATEGY: Building on and enhancing DOTS to meet the TB-related Millennium Development Goals. WHO/ HTM/TB/2006.368. World Health Organization, Geneva, Switzerland; 2006.

6. World Health Organization. The End TB Strategy. WHO/HTM/TB/2015.19. World Health Organization, Geneva, Switzerland; 2015.

7. World Health Organization. Global Tuberculosis Report 2016. WHO/HTM TB/2016. 13. World Health Organization, Geneva, Switzerland; 2016.

8. Dosso M, Bonnard D, Msellati P, Doulhourou C, Bamba A, Peyre M, et al. Surveillance des résistances secondaires aux anti-tuberculeux en Côte d'Ivoire 1995-1996. Int J Tuberc Lung Dis. 1999;3:805-9.

9. N'guessan K, Nahoua I, San Koffi M, Kouakou J, Dosso M. Primary resistance to antituberculosis drugs: trends in Côte d'Ivoire from 1996 to 2006. Med Mal Infect. 2008;38:231-2.

10. Hopprich R, Shephard L, Taing B, Kralj S, Smith A, Lumb R. Evaluation of (SD) MPT64 antigen rapid test, for fast and accurate identification of Mycobacterium tuberculosis complex. Pathology 2012;44:642-3.

11. Fabre M, Vong R, Gaillard T, Merens A, Gérome P, Saint-Blancard P, et al. Evaluation of the SD BIOLINE TB Ag MPT64 Rapid ${ }^{\circledR}$ for the diagnosis of tuberculosis. Pathol Biol. 2011;59:26-8.

12. Falzon D, Schünemann HJ, Harausz E, González-Angulo L, Lienhardt C, et al. World Health Organization treatment guidelines for drug-resistant tuberculosis, 2016 update. Eur Respir J. 2017;49. DOI: 10.1183/13993003.02308-2016.

13. Uplekar M, Weil D, Lonnroth K, Jaramillo E, Lienhardt C, Dias HM, et al. WHO's new End TB Strategy. Lancet 2015;385:1799-801.

14. Rigouts L, Gumusboga M, Bram de Rijk W, Nduwamahoro E, Uwizeye C, De J, et al. Mutations tuberculosis isolates with specific rpoB liquid culture system for Mycobacterium rifampin resistance missed in automated. J Clin Microbiol. 2013;51:2641-5.

15. Zignol M, Dean AS, Falzon D, Van Gemert W, Wright A, Van Deun A et al. Twenty years of Global Surveillance of Antituberculosis-Drug Resistance. N Engl J Med. 2016;375:1081-9. 